\title{
Epigenome-wide association study suggests that SNPs in the promoter region of $R E T N$ influence plasma resistin level via effects on DNA methylation at neighbouring sites
}

\author{
Masahiro Nakatochi ${ }^{1} \cdot$ Sahoko Ichihara ${ }^{2} \cdot$ Ken Yamamoto $^{3} \cdot$ Keizo Ohnaka $^{4}$. \\ Yosuke Kato $^{5}$ • Shigeki Yokota ${ }^{6}$ - Akihiro Hirashiki ${ }^{7}$ - Keiko Naruse ${ }^{8}$. \\ Hiroyuki Asano $^{6}$ - Hideo Izawa ${ }^{9}$ Tatsuaki Matsubara ${ }^{8}$ - Mitsuhiro Yokota ${ }^{10}$
}

Received: 19 May 2015 / Accepted: 28 August 2015 / Published online: 24 September 2015

(C) Springer-Verlag Berlin Heidelberg 2015

\begin{abstract}
Aims/hypothesis To investigate epigenetic regulation of the plasma concentration of resistin, we performed an epigenomewide association study for this variable and DNA methylation (DNAm) in an elderly Japanese cohort and then assessed the relation of single nucleotide polymorphisms (SNPs) associated with the plasma resistin concentration to DNAm level at identified sites.

Methods The association of plasma resistin level with DNAm status was examined in 191 nondiabetic elderly men with the Illumina Infinium HumanMethylation450 BeadChip array. The association between DNAm status at specific sites in the flanking region of the resistin gene (RETN) and RETN mRNA abundance was then evaluated with a public data set for 1202 monocyte samples from a multi-ethnic cohort. Finally, the association of DNAm status and SNPs in the promoter region of RETN was assessed in two cohorts comprising a total of 478 Japanese individuals.
\end{abstract}

Electronic supplementary material The online version of this article (doi:10.1007/s00125-015-3763-9) contains peer-reviewed but unedited supplementary material, which is available to authorised users.

Mitsuhiro Yokota

myokota@dpc.aichi-gakuin.ac.jp

1 Bioinformatics Section, Center for Advanced Medicine and Clinical Research, Nagoya University Hospital, Nagoya, Japan

2 Graduate School of Regional Innovation Studies, Mie University, Tsu, Japan

3 Department of Medical Chemistry, School of Medicine, Kurume University, Kurume, Japan

4 Department of Geriatric Medicine, Graduate School of Medical Sciences, Kyushu University, Fukuoka, Japan
Results DNAm status at cg02346997 located in the RETN promoter region showed a negative genome-wide significant association with the plasma resistin level $\left(p=6.02 \times 10^{-10}\right)$. Four DNAm sites in the RETN promoter region including $\operatorname{cg} 02346997\left(p=4.23 \times 10^{-70}\right)$ showed a negative genome-wide significant association with RETN mRNA abundance in monocytes. Furthermore, the number of minor alleles of the RETN promoter SNPs rs34861192 and rs3219175 was negatively associated with DNAm level at $\operatorname{cg} 02346997\left(p=4.43 \times 10^{-17}\right)$. Conclusions/interpretation Our results suggest that RETN promoter SNPs might influence the circulating resistin level through an effect on DNAm at $\operatorname{cg} 02346997$ and on RETN mRNA abundance in monocytes.

Keywords Cohort study · DNA methylation · Epigenetics · Epigenome-wide association study $\cdot$ Resistin $\cdot$ Single nucleotide polymorphism

5 Department of Registered Dietitians, Faculty of Health and Welfare, Tokai Gakuin University, Kakamigahara, Japan

6 Department of Internal Medicine, Iwakura Hospital, Iwakura, Japan

7 Department of Advanced Medicine in Cardiopulmonary Disease, Nagoya University Graduate School of Medicine, Nagoya, Japan

8 Department of Internal Medicine, School of Dentistry, Aichi Gakuin University, Nagoya, Japan

9 Department of Cardiology, Fujita Health University Banbuntane Hotokukai Hospital, Nagoya, Japan

10 Department of Genome Science, School of Dentistry, Aichi Gakuin University, 2-11 Suemori-dori, Chikusa-ku, Nagoya 464-8651, Japan 


$\begin{array}{ll}\text { Abbreviations } \\ \text { DNAm } & \text { DNA methylation } \\ \text { EWAS } & \text { Epigenome-wide association study } \\ \text { GLM } & \text { General linear model } \\ \text { IRI } & \begin{array}{l}\text { Fasting serum immunoreactive insulin } \\ \text { concentration }\end{array} \\ \text { KING } & \text { Kita-Nagoya Genomic Epidemiology } \\ \text { LD } & \text { Linkage disequilibrium } \\ \text { MESA } & \text { Multi-Ethnic Study of Atherosclerosis } \\ \text { SNP } & \text { Single nucleotide polymorphism }\end{array}$

\section{Introduction}

Resistin is an adipokine that is expressed predominantly in adipose tissue in mice, and its circulating concentration is markedly increased in genetic and diet-induced mouse models of obesity. The serum resistin level was also found to be reduced by treatment of mice with rosiglitazone, an insulinsensitising drug that interacts with peroxisome proliferatoractivated receptor $\gamma(\operatorname{PPAR} \gamma)$. Such observations have implicated resistin as a key player in obesity-related insulin resistance and type 2 diabetes mellitus [1].

Human resistin, on the other hand, is expressed predominantly in monocytes and macrophages, being rarely expressed in adipose tissue [2, 3]. Monocytes and macrophages are thus thought to be the main source of circulating resistin in humans. Human resistin is also considered to be a biomarker or mediator of metabolic and inflammatory diseases, with increased resistin levels having been associated with metabolic disorders such as obesity, insulin resistance, type 2 diabetes and atherosclerotic cardiovascular disease [4-8]. Recent prospective studies such as the Framingham Offspring Study and Multi-Ethnic Study of Atherosclerosis (MESA) thus found that the circulating resistin level was independently associated with the incidence of atherosclerosis $[9,10]$. Other studies, however, have found no significant association of the circulating resistin level with obesity, insulin resistance or cardiovascular events $[11,12]$. Identification of the determinants of the circulating resistin level will therefore be important for efforts to understand the contribution of resistin to disease and to prevent disease development.

Genetic factors contribute to the circulating concentration of resistin. A family-based study estimated that $\sim 70 \%$ of the observed variation in serum resistin levels was heritable in nondiabetic white people [13]. Candidate gene studies [14-17] and genome-wide association studies [18, 19] have been performed to identify single nucleotide polymorphisms (SNPs) that contribute to the circulating resistin concentration, with SNPs located around genes such as RETN, PPARG, $D C N, T Y W 3 / C R Y Z$ and NDST4 having been implicated. In particular, SNPs in the promoter region of the resistin gene $($ RETN) are thought to be a key determinant of the circulating resistin level. For example, rs1862513 in the RETN promoter was found to be associated with the plasma resistin concentration, accounting for $26.1 \%$ of the variance in this variable in a Japanese population [15]. We previously found that two SNPs - rs34861192 in the promoter region and rs3745368 in the $3^{\prime}$ untranslated region of RETN -independently contributed to the plasma resistin level in an aged Japanese population, with the combination of both SNPs accounting for 37.9\% of the variance in resistin concentration [14]. In addition, rs3219175 in the promoter region of RETN was found to be strongly associated with plasma resistin level, with rs3219175 and rs34861192 being shown to be in nearly complete linkage disequilibrium (LD) $[14,20]$. Although these latter two SNPs contribute to resistin levels to a greater extent than does rs1862513, the mechanism by which they regulate the circulating resistin concentration has remained unclear.

DNA methylation (DNAm) is an epigenetic modification that plays a role in the regulation of gene expression. About $23 \%$ of DNAm in blood cells has also been found to be heritable [21], and SNPs have been associated with differences in DNAm level [22, 23]. Furthermore, SNPs have been shown to influence mRNA levels through effects on DNAm [22-24]. The rs34861192 and rs3219175 SNPs in the RETN promoter might thus influence DNAm around RETN in monocytes and macrophages and thereby regulate resistin levels. The relations among circulating resistin level, DNAm status and SNPs have not been examined in a comprehensive manner to date, however.

We have now measured genome-wide DNAm levels for whole-blood DNA samples from the elderly Japanese participants in the Kita-Nagoya Genomic Epidemiology (KING) study and performed an epigenome-wide association study (EWAS) for plasma resistin level. With the use of a public data set, we then confirmed the association of RETN mRNA abundance in monocytes with methylation status at sites located in the flanking region of RETN. Finally, we assessed the association between DNAm sites identified in our study and SNPs in the flanking region of RETN with samples from two Japanese cohorts.

\section{Methods}

Study participants This study is a cross-sectional observational study designed to examine the association of methylation status at DNAm sites with plasma resistin level and is based on the ongoing KING study (ClinicalTrials.gov registration no. NCT00262691) [14, 25]. For the present study, 192 nondiabetic men were randomly selected. For the replication cohort in our examination of the association between DNAm levels and SNPs, we used samples from the general population-based Fukuoka cohort study [26, 27], with 287 participants (136 men, 151 women) being randomly 
selected. Detailed information on each cohort is provided in the electronic supplementary material (ESM) Methods, and the characteristics of the participants are shown in Table 1.

DNAm analysis Genome-wide DNAm profiles were obtained by analysis with an Infinium HumanMethylation 450 BeadChip (Illumina, San Diego, CA, USA). Details of the analysis are provided in ESM Methods.

Genotyping of RETN SNPs SNPs (rs34861192, rs1862513, rs3745368, rs3219175, rs3745367) located in the flanking region of RETN were genotyped for participants in the KING and Fukuoka studies. Genotyping methods are described in ESM Methods.

Profiles of mRNA and DNAm in publicly available data To confirm the association between RETN mRNA abundance and DNAm levels for nine DNAm sites in the flanking region of RETN in monocytes, we made use of publicly available data from the MESA Epigenomics and Transcriptomics study [28]. Details are provided in ESM Methods.

Statistical analysis Given that the distribution of plasma resistin level was skewed, the values were $\log _{10}$-transformed. DNAm level was quantified as an $M$ value, which can be converted to a $\beta$ value according to the equation: $\beta=2^{M} /\left(2^{M}+1\right)$ [29]. For the KING study data, the association of DNAm level for each DNAm site with plasma resistin level was assessed with a general linear model (GLM) with adjustment for age; the dependent variable was plasma resistin concentration and independent variables included the DNAm level for each site and age. To replicate the association of DNAm level for DNAm sites in the flanking region of RETN with plasma resistin concentration, we adopted a GLM with adjustment for age and sex for the Fukuoka study.

To test the association of DNAm level for sites located in the flanking region of RETN with RETN expression level in the MESA data set, we used a linear mixed-effects model based on the lmer function in lme4 of the R package [30], with fitting by maximum likelihood. The linear mixedeffects model was adjusted for both fixed effects (age) and random effects (RaceGenderSite). We applied a likelihood ratio test to assess the significance of the DNAm effect. The $p$ value for the DNAm effect in each model was calculated from the $\chi^{2}$ distribution with one degree of freedom and $-2 \times \log ($ likelihood ratio) as the test statistic.

To test the association of DNAm level at each DNAm site with each SNP for the KING study, we adopted a GLM with adjustment for age; the dependent variable was the DNAm level at each site and independent variables included the genotype of each SNP and age. We coded genotypes as 0,1 or 2, based on the number of copies of the minor allele. For the Fukuoka study, we adopted a GLM with adjustment for age and sex to test the association of DNAm level at each site with each SNP.

Additional statistical analyses were performed as described in ESM Methods.

For the EWAS analysis, the significance level $\alpha$ was determined by dividing 0.05 by the number of DNAm sites for Bonferroni correction $\left(\alpha=0.05 / 452,831=1.10 \times 10^{-7}\right)$. A $p$ value of $<0.05$ was considered nominally significant. All statistical analysis was performed with the R project (www. r-project.org) (version 3.1.3).
Table 1 Characteristics of the study participants

\begin{tabular}{llll}
\hline Characteristic & KING study $(n=192)$ & Fukuoka study $(n=287)$ & MESA study $(n=1202)$ \\
\hline Male, $n(\%)$ & $192(100 \%)$ & $136(47.4 \%)$ & $(49 \%)^{\mathrm{a}}$ \\
Age $($ years $)$ & $66(62,71)$ & $63(58,68)$ & $60(52,68)$ \\
BMI $\left(\mathrm{kg} / \mathrm{m}^{2}\right)$ & $23.2(21.3,24.5)$ & $23.2(21.2,25.1)$ & \\
Past or current smoker, $n(\%)$ & $129(67.2 \%)$ & $132(46.0 \%)$ & \\
$\mathrm{HbA}_{1 \mathrm{c}}(\%)^{\mathrm{b}}$ & $5.5(5.4,5.7)$ & $5.4(5.2,5.7)$ & \\
$\mathrm{HbA}_{1 \mathrm{c}}(\mathrm{mmol} / \mathrm{mol})$ & $36.4(35.3,38.5)$ & $35.3(33.2,38.5)$ & \\
${\text { Plasma resistin }(\mathrm{ng} / \mathrm{ml})^{\mathrm{c}}}_{\text {RETN mRNA level }}^{\mathrm{d}}$ & $9.4(6.6,13.9)$ & $6.3(5.1,9.0)$ & $392(298,522)$ \\
\hline
\end{tabular}

Continuous data are medians (1st quartile, 3rd quartile)

${ }^{a}$ The percentage of males in the MESA study is based on the value calculated for the 1,264 participants of the original study [28] because there is no sex-only information for the 1,202 participants in the publicly available data

${ }^{\mathrm{b}} \mathrm{HbA}_{1 \mathrm{c}}$ was measured with the Japan Diabetes Society (JDS) method and converted to NGSP (\%) and IFCC ( $\mathrm{mmol} / \mathrm{mol})$ units

${ }^{\mathrm{c}}$ Plasma resistin level was measured with the use of an ELISA kit for human resistin (LINCO Research for the KING study and Biovendor Laboratory Medicine for the Fukuoka study)

${ }^{\mathrm{d}}$ The level of RETN mRNA is presented as normalised signal intensity as measured by microarray analysis 


\section{Results}

Association analysis for DNAm level and plasma resistin concentration We performed genome-wide DNA methylation profiling for whole-blood DNA from 192 elderly nondiabetic men in the KING study. After initial processing, 191 individuals and 452,831 DNAm sites remained for subsequent analysis. We initially performed association analysis for DNAm level at each DNAm site and plasma resistin level. A single DNAm site (cg02346997) that achieved a genomewide significance level was detected on chromosome 19 (ESM Fig. 1). This site is located in the upstream region of RETN and was negatively associated with plasma resistin level (Table 2). It was also detected as an outlier in a quantilequantile (Q-Q) plot of $-\log _{10}(p)$ for the 452,831 tests of association between DNAm status and plasma resistin level (ESM Fig. 2). We next focused on the flanking region of RETN including cg02346997, ranging from 7733 to $7736 \mathrm{~kb}$ on chromosome 19 (Fig. 1a). Nine DNAm sites in this region are included on the Infinium HumanMethylation450 array. Among these nine DNAm sites, four sites are located in the upstream region of RETN, three in the body of the gene and two in the downstream region. Furthermore, three DNAm sites are located within a $\mathrm{CpG}$ island. Of the eight DNAm sites excluding cg02346997, two sites (cg21777015, cg22322184) showed nominally significant associations with plasma resistin level (Fig. 1b, Table 2). These two sites are located together with $\operatorname{cg} 02346997$ in the north $(\mathrm{N})$ shore region of the $\mathrm{CpG}$ island and were also negatively associated with plasma resistin level.

For the three DNAm sites significantly associated with plasma resistin level in the KING study, we evaluated these associations in 287 individuals in the Fukuoka study. Both cg02346997 and cg22322184 showed genome-wide significant associations with the plasma resistin concentration, and cg21777015 showed a nominally significant association (Table 2). The directions of these associations in the Fukuoka study were the same as those in the KING study.

We also re-evaluated the relations of the nine DNAm sites in the flanking region of RETN to plasma resistin level with adjustment for age, BMI, smoking status and cell type composition of the samples for the KING study. The same three DNAm sites (cg21777015, cg02346997, cg22322184) showed significant associations with plasma resistin level (ESM Table 1).

Association analysis for DNAm level and expression of $\boldsymbol{R E T N}$ in monocyte samples To examine the relation between DNAm status at the nine DNAm sites in the flanking region of RETN and the expression level of RETN in monocytes, we performed an association analysis with a data set derived from 1202 monocyte samples in the MESA study (Fig. 1c). The results for the MESA study were consistent with those for our KING study. Four DNAm sites ( $\operatorname{cg} 08132525$, cg21777015, cg02346997, cg22322184) achieved a genomewide level of significance, and three DNAm sites showed a nominally significant association (Table 3 ).

We inspected DNAm levels for the nine DNAm sites in the flanking region of RETN (Fig. 2a,b). Of these DNAm sites, three sites (cg21777015, cg02346997, cg22322184) showed moderate DNAm levels in both the KING and MESA data sets. Two other DNAm sites in the upstream region of RETN (cg20692181, cg08132525) showed a high level of DNAm, whereas the four DNAm sites in or downstream of the $\mathrm{CpG}$ island showed a low DNAm level. The levels of DNAm at the nine sites were positively correlated with each other (Fig. 2c, d).

Association analysis of DNAm level and SNPs We genotyped five SNPs in the flanking region of RETN (Fig. 1a) for the same samples of the KING study analysed with the
Table 2 Association analysis for plasma resistin level and DNAm sites in the flanking region of RETN for the KING and Fukuoka studies

\begin{tabular}{|c|c|c|c|c|c|c|}
\hline \multirow[t]{2}{*}{ DNAm site } & \multirow{2}{*}{$\begin{array}{l}\text { Position } \\
\text { on chr19 (bp) }\end{array}$} & \multirow{2}{*}{$\begin{array}{l}\text { Relation to } \\
\text { CpG island }\end{array}$} & \multicolumn{2}{|c|}{ KING study $(n=191)$} & \multicolumn{2}{|c|}{ Fukuoka study $(n=287)$} \\
\hline & & & Effect \pm SE & $p$ & Effect \pm SE & $p$ \\
\hline cg20692181 & $7,733,174$ & $\mathrm{~N}$ shore & $0.008 \pm 0.023$ & 0.735 & & \\
\hline $\operatorname{cg} 08132525$ & $7,733,401$ & $\mathrm{~N}$ shore & $-0.066 \pm 0.034$ & 0.055 & & \\
\hline $\operatorname{cg} 21777015$ & $7,733,793$ & $\mathrm{~N}$ shore & $-0.072 \pm 0.030$ & $0.017^{*}$ & $-0.095 \pm 0.025$ & $1.89 \times 10^{-4 *}$ \\
\hline $\operatorname{cg} 02346997$ & $7,733,883$ & $\mathrm{~N}$ shore & $-0.270 \pm 0.041$ & $6.02 \times 10^{-10 \dagger}$ & $-0.247 \pm 0.024$ & $5.29 \times 10^{-21 \dagger}$ \\
\hline $\operatorname{cg} 22322184$ & $7,734,203$ & $\mathrm{~N}$ shore & $-0.287 \pm 0.066$ & $2.08 \times 10^{-5 *}$ & $-0.294 \pm 0.038$ & $8.85 \times 10^{-14 \dagger}$ \\
\hline $\operatorname{cg} 06379095$ & $7,735,239$ & CpG island & $0.018 \pm 0.020$ & 0.378 & & \\
\hline $\operatorname{cg} 06411096$ & $7,735,294$ & $\mathrm{CpG}$ island & $-0.021 \pm 0.081$ & 0.797 & & \\
\hline $\operatorname{cg} 13380624$ & $7,735,426$ & $\mathrm{CpG}$ island & $0.005 \pm 0.022$ & 0.831 & & \\
\hline $\operatorname{cg} 06633066$ & $7,735,510$ & S shore & $-0.009 \pm 0.030$ & 0.772 & & \\
\hline
\end{tabular}

The Effect and $p$ values for the KING and Fukuoka studies were calculated with a GLM, with the Effect values representing change in $\log _{10}$ (plasma resistin level) per one unit change in $M$ value

*Nominal significance $(p<0.05)$; ${ }^{\dagger}$ genome-wide significance $\left(p<1.10 \times 10^{-7}\right)$

chr, chromosome; N, north; S, south 
Fig. 1 Genomic structure and regional plots for RETN. (a) Schematic representation of the genomic structure of RETN as well as of DNAm sites and SNPs of RETN evaluated in this study. White and black boxes represent untranslated regions and coding sequence, respectively. Genomic coordinates are based on UCSC hg19. (b, c) Regional plots of the flanking region of RETN for the KING and MESA studies, respectively. The vertical axis represents $-\log _{10}$ ( $p$ value) for assessment of the association of each methylation site either with plasma resistin level for the KING study (b) or with RETN mRNA abundance in monocytes for the MESA study (c). The DNAm sites that achieved a genome-wide significance level $\left(\alpha=1.10 \times 10^{-7}\right)$ are represented as black triangles, those that achieved a nominal significance level $(\alpha=0.05)$ are denoted with grey squares, and other DNAm sites are shown as white circles a

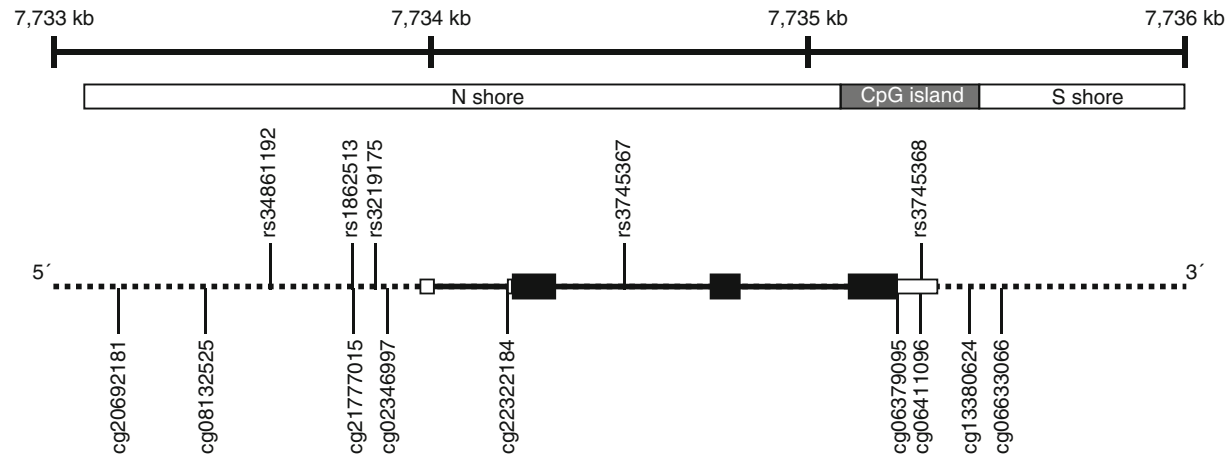

b
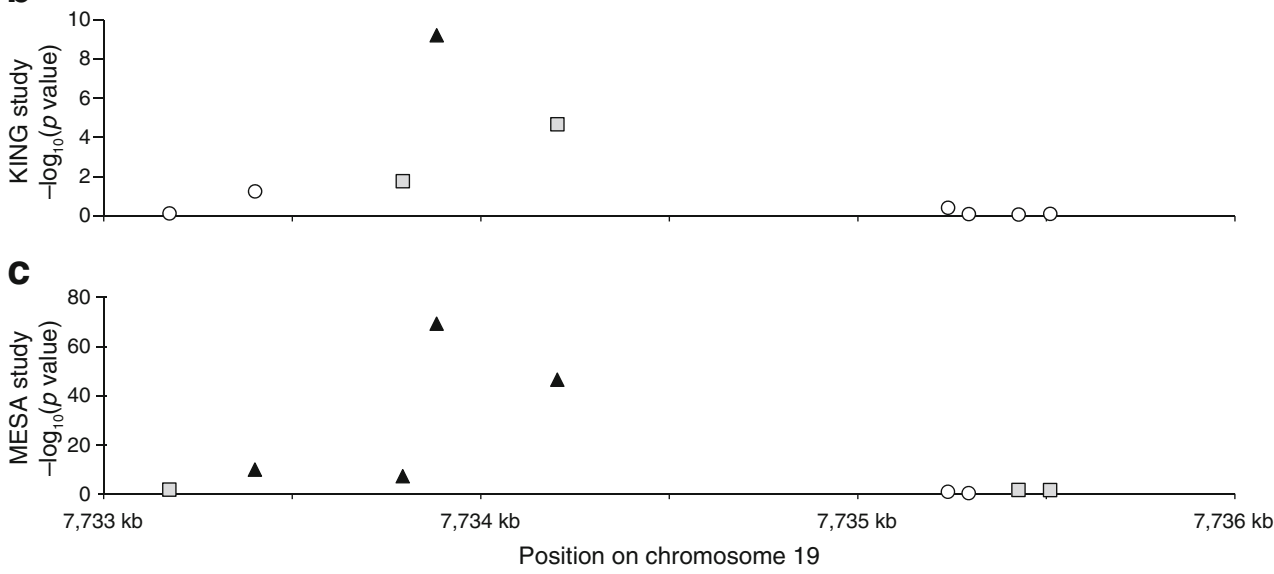

DNAm array. We then performed an association analysis for these five SNPs and DNAm level at the three DNAm sites found to be significantly associated with plasma resistin level in the KING study. Significant associations between each SNP and DNAm sites were detected (ESM Table 2). In particular, the SNPs rs34861192 and rs3219175 and the DNAm site $\operatorname{cg} 02346997$ showed the most significant associations
(Fig. 3a, ESM Table 2). These two SNPs (rs34861192, rs3219175) were in complete LD in our samples. The DNAm level at cg02346997 declined as the number of minor alleles for these SNPs increased.

We also evaluated the relation of rs3219175 to DNAm level at $\operatorname{cg} 02346997, \operatorname{cg} 21777015$ and $\operatorname{cg} 22322184$ in Fukuoka samples (ESM Table 2). A significant association
Table 3 Association analysis for RETN mRNA abundance in monocytes and DNAm sites in the flanking region of RETN for the MESA study

\begin{tabular}{lllll}
\hline DNAm site & Position on chr19 $(\mathrm{bp})$ & Relation to CpG island & \multicolumn{2}{l}{ MESA study $(n=1202)$} \\
\cline { 4 - 5 } & & & Effect \pm SE & $p$ \\
\hline $\operatorname{cg} 20692181$ & $7,733,174$ & N shore & $-0.080 \pm 0.035$ & $0.021^{*}$ \\
$\operatorname{cg} 08132525$ & $7,733,401$ & N shore & $-0.217 \pm 0.034$ & $1.40 \times 10^{-10^{\dagger}}$ \\
$\operatorname{cg} 21777015$ & $7,733,793$ & N shore & $-0.108 \pm 0.020$ & $7.14 \times 10^{-8 \dagger}$ \\
$\operatorname{cg} 02346997$ & $7,733,883$ & N shore & $-0.298 \pm 0.016$ & $4.23 \times 10^{-70 \dagger}$ \\
$\operatorname{cg} 22322184$ & $7,734,203$ & N shore & $-0.363 \pm 0.024$ & $2.97 \times 10^{-47 \dagger}$ \\
$\operatorname{cg} 06379095$ & $7,735,239$ & CpG island & $-0.035 \pm 0.025$ & 0.151 \\
$\operatorname{cg} 06411096$ & $7,735,294$ & CpG island & $0.023 \pm 0.037$ & 0.540 \\
$\operatorname{cg} 13380624$ & $7,735,426$ & CpG island & $-0.055 \pm 0.025$ & $0.028^{*}$ \\
$\operatorname{cg} 06633066$ & $7,735,510$ & S shore & $0.065 \pm 0.030$ & $0.032^{*}$ \\
\hline
\end{tabular}

The Effect and $p$ values were calculated with a linear mixed-effects model, with the Effect values representing change in $\log _{10}$ (RETN expression level) per one unit change in $M$ value *Nominal significance $(p<0.05) ;{ }^{\dagger}$ genome-wide significance $\left(p<1.10 \times 10^{-7}\right)$ chr, chromosome; N, north; S, south 
Fig. 2 DNAm levels for nine DNAm sites in the vicinity of RETN for the KING and MESA studies. (a, b) The box-andwhisker plots are based on the KING study $(n=191)$ (a) and the MESA study $(n=1,202)(\mathbf{b})$, with the order of the sites being based on their position. Data were converted from $M$ values to $\beta$ values. (c, d) Correlation heat maps for DNAm levels at the nine sites based on $M$ values in the KING and MESA studies, respectively. The $r$ value represents Pearson's correlation coefficient

Fig. 3 DNAm level at cg02346997 according to genotype for rs3219175 in the KING and Fukuoka studies. The box-and-whisker plots are based on the KING study ( $n=191)$ (a) and the Fukuoka study $(n=287)$ (b). Data were converted from $M$ values to $\beta$ values. The $p$ values were $4.43 \times 10^{-17}$ (a) and $3.50 \times$ $10^{-19}(\mathbf{b})$ and were calculated with a GLM based on $M$ value with adjustment for age (a) or age and sex (b) a

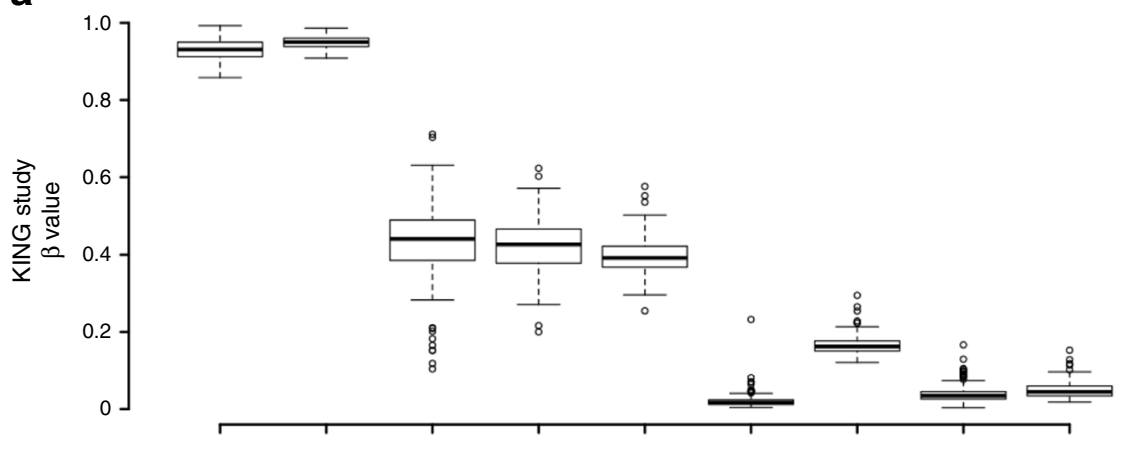

b

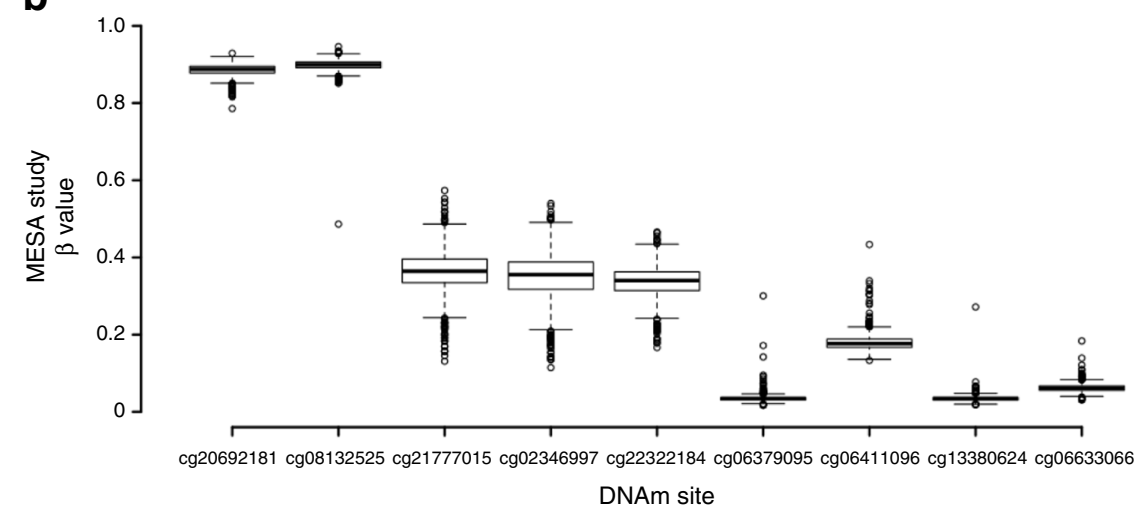

C d
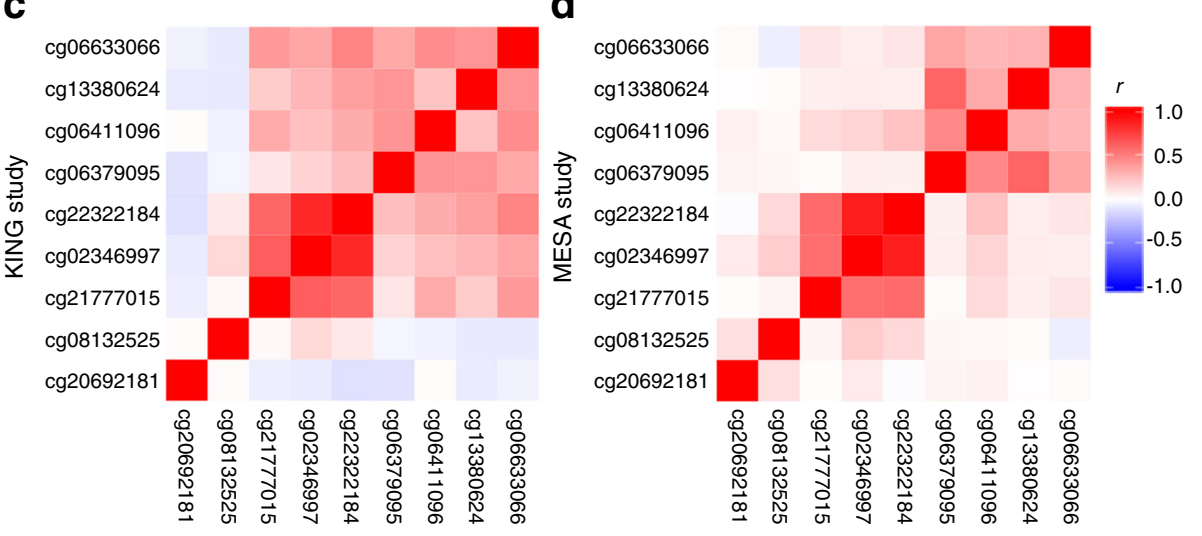

a

b

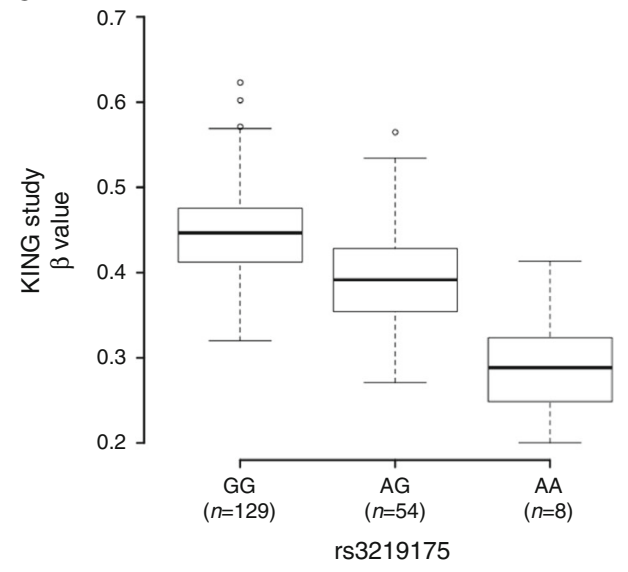


between this SNP and $\operatorname{cg} 02346997$ was replicated in the Fukuoka samples (Fig. 3b, ESM Table 2).

Association analysis of plasma resistin concentration, SNPs and DNAm level To compare the independent contributions of the SNP rs3219175 and DNAm level at cg02346997 with plasma resistin level in the KING study, we calculated the contribution rate $\left(R^{2}\right)$ based on the partial correlation coefficient. Both $\operatorname{cg} 02346997\left(R^{2}=0.029, p=\right.$ $0.017)$ and $\mathrm{rs} 3219175\left(R^{2}=0.273, p=4.56 \times 10^{-17}\right)$ showed a significant $R^{2}$ value. Although the contribution of rs 3219175 to the plasma resistin level was greater than that of cg02346997, the DNAm site also clearly affected plasma resistin concentration.

Finally, we investigated the relation of plasma resistin level, the SNP rs3219175, and the DNAm sites cg02346997 and cg22322184 to type 2 diabetes-related traits with the use of a GLM with adjustment for age, BMI and smoking status for the KING study (ESM Table 3). However, no significant associations were detected.

\section{Discussion}

We have here performed an EWAS for plasma resistin level in participants of the KING study with the use of the Infinium HumanMethylation450 array. Our analysis of 191 elderly Japanese men detected a genome-wide significant association of plasma resistin level with one DNAm site (cg02346997) located in the promoter region of RETN. We then focused our association analysis on the flanking region of RETN and found nominally significant associations of plasma resistin concentration with two additional DNAm sites (cg21777015, cg22322184) in the promoter region and $5^{\prime}$ untranslated region of RETN. A replication study of the relation between plasma resistin level and DNAm sites revealed genome-wide significant associations of cg02346997 and $\operatorname{cg} 22322184$ as well as a nominally significant association of $\operatorname{cg} 21777015$ in the Fukuoka study. A study of the relation between RETN mRNA abundance and DNAm sites in the flanking region of $R E T N$ with a publicly available data set for monocyte samples from the MESA study revealed genome-wide significant associations of $\operatorname{cg} 02346997, \operatorname{cg} 22322184$ and $\operatorname{cg} 21777015$ with RETN mRNA level in monocytes. Furthermore, we found that DNAm status for these three DNAm sites was associated with SNPs located in the promoter region of RETN in two Japanese cohorts, those of the KING and Fukuoka studies. In particular, the SNPs rs34861192 and rs3219175 and DNAm status at $\operatorname{cg} 02346997$ showed the most significant associations. The DNAm level at cg02346997 was thus negatively associated with plasma resistin level and RETN mRNA abundance in monocytes, and it decreased as the number of minor alleles for rs34861192 and rs3219175 increased. The circulating resistin level was previously found to increase as the number of minor alleles for these SNPs increased $[14,20]$. Our results thus suggest that a causal relation between the plasma resistin level and SNPs in the promoter region of RETN is mediated via changes in DNAm in this region and in RETN transcription in monocytes.

The circulating concentration of resistin has previously been associated with SNPs in the promoter region of RETN including rs34681192, rs3219175 and rs1862513. These SNPs are located in the same LD block in the Japanese population [20], with rs34861192 and rs3219175 being in nearly complete LD [14, 20]. Among these three SNPs, rs 1862513 was previously found to affect RETN promoter activity [31] and rs3219175 was suggested to influence the binding of proteins such as nuclear factor (NF)-KB [20]. However, the detailed mechanism by which rs34861192 and rs3219175 might affect plasma resistin level has remained unclear. The DNAm site cg02346997, found to be significantly associated with plasma resistin level in our study, is located only $28 \mathrm{bp}$ downstream of the SNP rs3219175 (Fig. 1a), consistent with the notion that DNAm level at cg02346997 might be affected by rs3219175. Our results thus suggest that rs3219175 and rs34861192 might regulate plasma resistin level by influencing DNAm at cg02346997 and RETN transcription in monocytes.

We found that DNAm sites in a CpG island shore such as cg02346997, cg22322184 and cg21777015 were more significantly associated with plasma resistin level than were DNAm sites within the $\mathrm{CpG}$ island itself such as $\mathrm{cg} 06379095$, cg06411096 and $\operatorname{cg} 13380624$ (Fig. 1). A CpG island shore is defined as a $2000 \mathrm{bp}$ region upstream ( $\mathrm{N}$ shore) or downstream ( $\mathrm{S}$ shore) of a $\mathrm{CpG}$ island [32]. Most differences in DNAm among normal tissues, or between normal and cancer tissues, have been found to occur in $\mathrm{CpG}$ island shores rather than in the islands themselves [32]. Our results are thus consistent with this finding, and they indicate that DNAm sites in the $\mathrm{N}$ shore of the $\mathrm{CpG}$ island of RETN, rather than those within the island itself, are functional with regard to regulation of circulating resistin level.

To identify potential monocyte-specific functional regions of RETN and its flanking region, we examined CTCF binding sites, histone modifications and DNase I peaks previously described for a human monocyte (CD14 $\left.{ }^{+}\right)$sample [33] (ESM Fig. 3). The region including DNAm sites cg02346997, cg21777015 and cg22322184 as well as SNPs rs34861192 and rs3219175 overlaps with a region characterised by DNase I hypersensitivity [34] and a high level of histone modification ( $\mathrm{H} 3 \mathrm{~K} 4 \mathrm{~m} 1 / 2 / 3, \mathrm{H} 3 \mathrm{~K} 27 \mathrm{ac})$, which are indicators of a potential regulatory region. Binding sites for the transcription factors c-Rel, CCAAT/enhancer binding protein $\alpha$ $(\mathrm{C} / \mathrm{EBP} \alpha)$, activating transcription factor 2 (ATF2) and activator protein (AP1) have been identified within a 619 bp region upstream of the translation start site of RETN [35]. The DNAm site $\operatorname{cg} 02346997$ is located within this region. The 
transcription factor Sp1 was also found to play an important role in RETN transcription, with a predicted Sp1 binding site also being located near cg02346997 [35]. It is thus possible that DNAm at $\operatorname{cg} 02346997$ regulates RETN transcription by preventing the binding of these transcription factors to DNA.

Variation in DNAm level at cg02346997 might be affected by SNPs within \pm 1 bp of this site. Inspection of dbSNP build 142 revealed the SNP rs 575656735 at 7,733,883 bp on chromosome 19, which is the same position as $\operatorname{cg} 02346997$. This SNP has major and minor alleles of $\mathrm{C}$ and $\mathrm{T}$, respectively, and was reported by the Genome of the Netherlands (GoNL) project $[36,37]$, which aims to characterise DNA sequence variation in the Dutch population. The website of this project (www.nlgenome.nl) states that the frequency of the minor $\mathrm{T}$ allele of rs 575656735 is low $(0.1 \%)$ in the Dutch population. Furthermore, this SNP was not reported in the 1000 Genomes Project, which includes Japanese samples. It is therefore likely that this SNP does not contribute to the variation in DNAm at $\operatorname{cg} 02346997$ in Japanese individuals. Both rs545198984 and rs143039347 are located within \pm 1 bp of cg22322184, but these SNPs were not reported in Asian samples of the 1000 Genomes Project, suggesting that they also do not contribute to the variation in DNAm at cg22322184 in Japanese people.

The frequency of the minor A allele of rs3219175 was found to be high in Africans (12.9\%) and East Asians $(17.9 \%)$, but relatively low in Americans (1.2\%), Europeans (1.0\%) and South Asians (2.4\%), in phase III of the 1000 Genomes Project. The SNPs rs3219175 and rs34861192 might thus be expected to contribute to the circulating resistin level in other East Asian and African populations in addition to Japanese. Indeed, rs34861192 and rs3219175 were found to be associated with the plasma resistin concentration, accounting for $13.3 \%$ and $12.8 \%$ of the variance in this variable, respectively in a homogeneous Malay population in Malaysia [38]. Our results suggest that these associations might also be mediated via changes in DNAm at $\operatorname{cg} 02346997$ and cg22322184. Furthermore, we detected a similar pattern of association between DNAm and either plasma resistin level or RETN expression in the KING and MESA data sets, which are derived from Japanese and multiple ethnic groups (whites, African-Americans, Hispanics) [28], respectively. This similarity in the association pattern might thus be due to the presence of African-American samples with a higher frequency of the A allele of rs3219175 in the MESA cohort.

Resistin has been implicated as a key player in obesityrelated insulin resistance and type 2 diabetes [1]. We previously investigated the relations of the plasma resistin concentration and the SNP rs34861192 to type 2 diabetes and type 2 diabetes-related traits in 3133 Japanese individuals from the KING study, and we found that the plasma resistin level was positively associated with the fasting serum immunoreactive insulin concentration (IRI) and HOMA-IR [14]. The contribution rates of IRI and HOMA-IR to the plasma resistin level were low $\left(R^{2}=0.005\right.$ and 0.003 , respectively), however [14]. In addition, there was no significant association of rs34861192 with type 2 diabetes-related traits. In the present study, we examined the association of plasma resistin level, the SNP rs34861192, and the DNAm sites cg02346997 and cg22322184 with type 2 diabetes-related traits for the KING study participants. However, we detected no significant associations, possibly as a result of insufficient power due to the small sample size (the sample sizes required to detect contribution rates of 0.005 and 0.003 for the correlation analysis are 1566 and 2613, respectively, assuming $\alpha=0.05$ and power $=0.8$ ). Further population-based association studies with larger sample sizes are thus required to elucidate the relations of circulating resistin level and cg02346997 and cg22322184 to type 2 diabetes and related traits.

With regard to limitations of the present study, the EWAS was performed with the Infinium HumanMethylation450 array, with the consequence that not all DNAm sites in the vicinity of RETN were inspected. Further insight into regulation of RETN expression by DNAm will require finemapping analysis with bisulfite sequencing. In addition, the present study is cross-sectional in nature and therefore does not establish a cause-and-effect relation between DNAm level at DNAm sites and plasma resistin concentration. Future studies are thus necessary to evaluate such relations in prospective cohorts. Finally, plasma resistin level might be influenced by post-transcriptional mechanisms, with the association between RETN mRNA abundance and plasma resistin level thus still requiring confirmation in the KING or Fukuoka studies.

In conclusion, we have demonstrated an association of DNAm level at $\operatorname{cg} 02346997$ with plasma resistin level and RETN mRNA abundance in monocytes. Furthermore, we found that DNAm level at this site was associated with the SNPs rs34861192 and rs3219175 located in the promoter region of RETN. Our results thus suggest the possibility that plasma resistin level might be influenced by SNPs in the promoter region of RETN via changes in DNAm in this region and in RETN mRNA abundance in monocytes.

\section{Acknowledgements We thank all participants in this study.}

Funding This study was supported in part by Grants-in-Aid from the Ministry of Education, Culture, Sports, Science and Technology of Japan, including those for Research Activity Start-up (25893087) and Young Scientists (B) (15 K19242) to MN, for Scientific Research (B) to MY (24390169) and to TM (25293144), for Scientific Research (C) to KY (15 K08290), and for Funding Program for Next Generation WorldLeading Researchers (NEXT Program) to SI (LS056). It was also supported in part by Grants-in-Aid from the Ichihara International Scholarship Foundation to MN and from the Suzuken Memorial Foundation to MY (14-003).

Duality of interest The authors declare that there is no duality of interest associated with this manuscript. 
Contribution statement $\mathrm{MN}$ and MY designed the study, with contributions from SI, KY, YK, SY, AH, KN, HA, HI and TM. KY performed the experiments. SI, KO, AH, HA, HI and MY contributed to sample collection. MN analysed the data, with a contribution from KY. All authors interpreted and discussed the data. MN and MY wrote the manuscript, with all authors contributing to its critical review and revision, and to approval of the final version. MY is responsible for the integrity of this work as a whole.

\section{References}

1. Steppan CM, Bailey ST, Bhat S et al (2001) The hormone resistin links obesity to diabetes. Nature 409:307-312

2. Patel L, Buckels AC, Kinghorn IJ et al (2003) Resistin is expressed in human macrophages and directly regulated by PPAR gamma activators. Biochem Biophys Res Commun 300:472-476

3. Savage DB, Sewter CP, Klenk ES et al (2001) Resistin/Fizz3 expression in relation to obesity and peroxisome proliferator-activated receptor-gamma action in humans. Diabetes 50:2199-2202

4. Reilly MP, Lehrke M, Wolfe ML, Rohatgi A, Lazar MA, Rader DJ (2005) Resistin is an inflammatory marker of atherosclerosis in humans. Circulation 111:932-939

5. Fujinami A, Obayashi H, Ohta K et al (2004) Enzyme-linked immunosorbent assay for circulating human resistin: resistin concentrations in normal subjects and patients with type 2 diabetes. Clin Chim Acta 339:57-63

6. Lazar MA (2007) Resistin- and obesity-associated metabolic diseases. Horm Metab Res 39:710-716

7. Ohmori R, Momiyama Y, Kato R et al (2005) Associations between serum resistin levels and insulin resistance, inflammation, and coronary artery disease. J Am Coll Cardiol 46:379-380

8. McTernan CL, McTernan PG, Harte AL, Levick PL, Barnett AH, Kumar S (2002) Resistin, central obesity, and type 2 diabetes. Lancet 359:46-47

9. Rienstra M, Sun JX, Lubitz SA et al (2012) Plasma resistin, adiponectin, and risk of incident atrial fibrillation: the Framingham Offspring Study. Am Heart J 163:119-124 e111

10. Muse ED, Feldman DI, Blaha MJ et al (2015) The association of resistin with cardiovascular disease in the Multi-Ethnic Study of Atherosclerosis. Atherosclerosis 239:101-108

11. Utzschneider KM, Carr DB, Tong J et al (2005) Resistin is not associated with insulin sensitivity or the metabolic syndrome in humans. Diabetologia 48:2330-2333

12. Lee JH, Chan JL, Yiannakouris N et al (2003) Circulating resistin levels are not associated with obesity or insulin resistance in humans and are not regulated by fasting or leptin administration: cross-sectional and interventional studies in normal, insulin-resistant, and diabetic subjects. J Clin Endocrinol Metab 88:4848-4856

13. Menzaghi C, Coco A, Salvemini L et al (2006) Heritability of serum resistin and its genetic correlation with insulin resistance-related features in nondiabetic Caucasians. J Clin Endocrinol Metab 91:27922795

14. Asano H, Izawa H, Nagata K et al (2010) Plasma resistin concentration determined by common variants in the resistin gene and associated with metabolic traits in an aged Japanese population. Diabetologia 53:234-246

15. Osawa H, Tabara Y, Kawamoto R et al (2007) Plasma resistin, associated with single nucleotide polymorphism -420 , is correlated with insulin resistance, lower HDL cholesterol, and high-sensitivity Creactive protein in the Japanese general population. Diabetes Care 30:1501-1506

16. Onuma H, Tabara Y, Kawamura R et al (2013) Plasma resistin is associated with single nucleotide polymorphisms of a possible resistin receptor, the decorin gene, in the general Japanese population. Diabetes 62:649-652

17. Osawa H, Tabara Y, Kawamoto R et al (2009) PPARgamma Pro12Ala Pro/Pro and resistin SNP-420 G/G genotypes are synergistically associated with plasma resistin in the Japanese general population. Clin Endocrinol 71:341-345

18. Chung CM, Lin TH, Chen JW et al (2014) Common quantitative trait locus downstream of RETN gene identified by genome-wide association study is associated with risk of type 2 diabetes mellitus in Han Chinese: a Mendelian randomization effect. Diabetes Metab Res Rev 30:232-240

19. Qi Q, Menzaghi C, Smith S et al (2012) Genome-wide association analysis identifies TYW3/CRYZ and NDST4 loci associated with circulating resistin levels. Hum Mol Genet 21:4774-4780

20. Onuma H, Tabara Y, Kawamura R et al (2010) A at single nucleotide polymorphism-358 is required for $\mathrm{G}$ at -420 to confer the highest plasma resistin in the general Japanese population. PLoS One 5, e9718

21. Boks MP, Derks EM, Weisenberger DJ et al (2009) The relationship of DNA methylation with age, gender and genotype in twins and healthy controls. PLoS One 4, e6767

22. Gutierrez-Arcelus M, Lappalainen T, Montgomery SB et al (2013) Passive and active DNA methylation and the interplay with genetic variation in gene regulation. Elife 2, e00523

23. Bell JT, Pai AA, Pickrell JK et al (2011) DNA methylation patterns associate with genetic and gene expression variation in HapMap cell lines. Genome Biol 12:R10

24. Olsson AH, Volkov P, Bacos K et al (2014) Genome-wide associations between genetic and epigenetic variation influence mRNA expression and insulin secretion in human pancreatic islets. PLoS Genet 10, e1004735

25. Nakatochi M, Miyata S, Tanimura D et al (2011) The ratio of adiponectin to homeostasis model assessment of insulin resistance is a powerful index of each component of metabolic syndrome in an aged Japanese population: results from the KING Study. Diabetes Res Clin Pract 92:e61-e65

26. Nanri A, Yoshida D, Yamaji T, Mizoue T, Takayanagi R, Kono S (2008) Dietary patterns and C-reactive protein in Japanese men and women. Am J Clin Nutr 87:1488-1496

27. Yoshida D, Toyomura K, Fukumoto J et al (2009) Waist circumference and cardiovascular risk factors in Japanese men and women. J Atheroscler Thromb 16:431-441

28. Reynolds LM, Taylor JR, Ding J et al (2014) Age-related variations in the methylome associated with gene expression in human monocytes and T cells. Nat Commun 5:5366

29. Du P, Zhang X, Huang CC et al (2010) Comparison of Beta-value and $\mathrm{M}$-value methods for quantifying methylation levels by microarray analysis. BMC Bioinforma 11:587

30. Bates D, Maechler M, Bolker B, Walker S (2014) lme4: Linear mixed-effects models using Eigen and S4. Available from http:// CRAN.R-project.org/package=lme4. Accessed $22 \mathrm{Feb} 2015$

31. Osawa H, Yamada K, Onuma $\mathrm{H}$ et al (2004) The G/G genotype of a resistin single-nucleotide polymorphism at -420 increases type 2 diabetes mellitus susceptibility by inducing promoter activity through specific binding of Sp1/3. Am J Hum Genet 75:678-686

32. Irizarry RA, Ladd-Acosta C, Wen B et al (2009) The human colon cancer methylome shows similar hypo- and hypermethylation at conserved tissue-specific CpG island shores. Nat Genet 41:178186

33. Rosenbloom KR, Sloan CA, Malladi VS et al (2013) ENCODE data in the UCSC genome browser: year 5 update. Nucleic Acids Res 41: D56-D63

34. Crawford GE, Holt IE, Whittle J et al (2006) Genome-wide mapping of DNase hypersensitive sites using massively parallel signature sequencing (MPSS). Genome Res 16:123-131 
35. Singh AK, Battu A, Mohareer K, Hasnain SE, Ehtesham NZ (2010) Transcription of human resistin gene involves an interaction of Sp1 with peroxisome proliferator-activating receptor gamma (PPARgamma). PLoS One 5, e9912

36. Boomsma DI, Wijmenga C, Slagboom EP et al (2014) The genome of the Netherlands: design, and project goals. Eur J Hum Genet 22: 221-227
37. Genome of the Netherlands C (2014) Whole-genome sequence variation, population structure and demographic history of the Dutch population. Nat Genet 46:818-825

38. Apalasamy YD, Rampal S, Salim A et al (2015) Polymorphisms of the resistin gene and their association with obesity and resistin levels in Malaysian Malays. Biochem Genet 53:120-131 